\title{
Optimized Agroinfiltration and Virus-Induced Gene Silencing to Study Ve1-Mediated Verticillium Resistance in Tobacco
}

\author{
Zhao Zhang, ${ }^{1,2}$ Emilie Fradin, ${ }^{1,3}$ Ronnie de Jonge, ${ }^{1}$ H. Peter van Esse, ${ }^{1}$ Patrick Smit, ${ }^{1}$ Chun-Ming Liu, ${ }^{2}$ \\ and Bart P. H. J. Thomma ${ }^{1,3}$ \\ ${ }^{1}$ Laboratory of Phytopathology, Wageningen University, Droevendaalsesteeg 1, 6708 PB Wageningen, The Netherlands; \\ ${ }^{2}$ Key Laboratory of Plant Molecular Physiology, Institute of Botany, the Chinese Academy of Sciences, Beijing 100093, China; \\ ${ }^{3}$ Centre for BioSystems Genomics, P.O. Box 98, 6700 AB Wageningen, The Netherlands
}

Submitted 20 June 2012. Accepted 7 September 2012.

\begin{abstract}
Recognition of pathogen effectors by plant immune receptors often leads to the activation of a hypersensitive response (HR), which is a rapid and localized cell death of plant tissue surrounding the site at which recognition occurs. Due to its particular amenability to transient assays for functional genetics, tobacco is a model for immune signaling in the Solanaceae plant family. Here, we show that coexpression of the tomato (Solanum lycopersicum) immune receptor Ve1 and the corresponding Verticillium effector protein Ave1 leads to HR only in particular tobacco species. Whereas HR is obtained in Nicotiana tabacum, no such response is obtained in N. benthamiana. Furthermore, our analysis revealed an endogenous Ve1 ortholog in Nicotiana glutinosa, as expression of Ave1 in absence of Ve1 induced a $\mathrm{HR}$, and $N$. glutinosa was found to be resistant against race 1 Verticillium dahliae. We furthermore report the establishment of virus-induced gene silencing in N. tabacum for functional analysis of Ve1 signaling. Collectively, our data show that $N$. tabacum can be used as a model plant to study Ve1mediated immune signaling.
\end{abstract}

Verticillium dahliae and Verticillium albo-atrum are among the world's most notorious plant pathogens, occurring in temperate and subtropical regions and causing vascular wilt diseases in over 200 herbaceous and woody plant species (Fradin and Thomma 2006; Klosterman et al. 2009). Genetic resistance has been identified in several plant species, and a single dominant locus that confers Verticillium resistance named Ve has been used by tomato (Solanum lycopersicum) breeders for over 60 years. Isolates of Verticillium spp. that are contained by the Ve locus are assigned to race 1, while all others are designated as race 2 (Fradin and Thomma 2006). However, race 2 strains are typically not as aggressive as race 1 strains (Armen and Shoemaker 1985; de Jonge et al. 2012; Paternotte and van Kesteren 1993). The Ve locus comprises two closely linked inversely oriented genes, $\mathrm{Vel}$ and $\mathrm{Ve} 2$, which encode extracellular leucine-rich repeat (eLRR) receptor-like proteins (RLP)

Corresponding author: Bart P. H. J. Thomma; Telephone: +1 0031-317484536; Fax: +1 0031-317-483412; E-mail: bart.thomma@wur.nl

* The $\boldsymbol{e}$-Xtra logo stands for "electronic extra" and indicates that four supplementary figures are published online.

(C) 2013 The American Phytopathological Society
(Kawchuk et al. 2001). These are cell surface receptors that typically comprise an eLRR domain, a single-pass transmembrane domain, and a short cytoplasmatic tail that lacks obvious motifs for intracellular signaling. More recent research has demonstrated that only Vel acts as a functional Verticillium resistance gene in tomato, while no functionality could be assigned to Ve2 (Fradin et al. 2009, 2011).

Genes encoding RLP-type immune receptors were found to act in pathogen defense in several plant species (Fritz-Laylin et al. 2005; Wang et al. 2008, 2010). The interaction between Cladosporium fulvum and tomato that carries $C f$ resistance genes has been exploited extensively to study RLP-mediated resistance signaling (Thomma et al. 2005; Wulff et al. 2009). Based on their involvement in defense responses mediated by other RLP, candidate genes were tested for a role in Ve1-mediated Verticillium resistance in tomato, using virus-induced gene silencing (VIGS) (Fradin et al. 2009; 2011; Vossen et al. 2010). Despite the tools and resources that are available, research on tomato has major limitations when compared with other model plant species, such as the Solanaceous sister species Nicotiana benthamiana and the Brassicaceous model plant Arabidopsis thaliana, which are also genuine hosts for Verticillium infection. For instance, VIGS efficiency in tomato is not as good as in N. benthamiana (Liu et al. 2002). Interestingly, tomato $\mathrm{Vel}$ remains fully functional after transfer to Arabidopsis, suggesting that this model plant can be used to unravel the genetics of resistance signaling mediated by Ve1. Indeed, after discovering that functionality of the receptor-like kinase SERK1 is required for Ve1 signaling in Arabidopsis, the requirement of SERK1 for Ve1-mediated resistance was confirmed in tomato as well (Fradin et al. 2011).

To study tomato RLP, the model plant $N$. benthamiana (Goodin et al. 2008) has frequently been used for Agrobacterium transient transformation assay (ATTA) and VIGS (Bar and Avni 2009; Fradin et al. 2009; Gabriëls et al. 2006; Rowland et al. 2005; Van der Hoorn et al. 2000; Vossen et al. 2010). Since tomato and $N$. benthamiana are close relatives they share a high degree of coding sequence homology, allowing the use of tomato sequences to target homologs in $N$. benthamiana and vice versa (Faino et al. 2012; Gabriëls et al. 2006; Senthil-Kumar et al. 2007). So far, however, full exploitation of these assays for the investigation of Ve1-mediated signaling was hampered by the unknown identity of the Verticillium avirulence molecule that activates the Vel receptor. Recently, through comparative genomics of race 1 and race 2 strains, this molecule was identified, and named Ave1 (for

182 / Molecular Plant-Microbe Interactions 
avirulence on Ve1 tomato) (de Jonge et al. 2012). The availability of the tomato resistance gene $(\mathrm{Vel})$ and the corresponding $V$. dahliae avirulence gene (Ave1) facilitates studies into the genetics of Ve1-mediated Verticillium resistance. Here, we describe the development of protocols to investigate Ve1-mediated defense signaling in tobacco.

\section{RESULTS}

Coexpression of Ave1 and $\mathrm{Vel}$ induces a hypersensitive response (HR) in Nicotiana tabacum, but not in Nicotiana benthamiana.

Often, recognition of pathogen avirulence molecules by plant immune receptors leads to the activation of a HR, rapid and localized cell death of plant tissue at the site at which recognition occurs. For Ave1, it was shown that transient expression mediated by Potato virus X (PVX) (Chapman et al. 1992) specifically induced HR on tomato carrying Vel (de Jonge et al. 2012). To test whether a HR can also be induced in tobacco, coexpression of Avel and Vel by agroinfiltration was pursued. To this end, the coding sequence of Verticillium dahliae Ave1 was cloned into the Gateway-compatible vector pFAST-R02 (Shimada et al. 2010), generating expression construct pFAST::Avel to establish Avel expression driven by the Cauliflower mosaic virus (CaMV) $35 \mathrm{~S}$ promoter, and was transformed into Agrobacterium tumefaciens GV3101. The resulting strain was mixed in a $1: 1$ ratio with $A$. tumefaciens GV3101 carrying $p M O G 800:: V e 1$ to establish Vel expression similarly driven by the CaMV $35 \mathrm{~S}$ promoter (Fradin et al. 2009) and was infiltrated into the leaves of Nicotiana tabacum cv. Petite Havana SR1. At 1 to 2 days postinfiltration (dpi), leaf tissue started to collapse and the infiltrated leaves developed clear necrosis by 5 dpi (Fig. 1A). In contrast, agroinfiltration of $p M O G 800:: V e 1$ or $p F A S T:: A v e 1$ alone did not induce necrosis. Nevertheless, the HR that was induced upon coexpression of Vel and Avel was not as strong as HR induced upon coexpression of the corresponding tomato $C f-9$ and $C$. fulvum Avr9 gene pair, for which the complete infiltrated sectors became fully necrotic (Fig. 1B).

It has been suggested that the accumulation of transiently expressed protein usually peaks between 1 and 3 dpi and rapidly decreases thereafter, due to post-transcriptional gene silencing (PTGS) in the host plant (Johansen et al. 2001; Van der Hoorn et al. 2003; Voinnet et al. 2003). Thus, weak HR induced upon co-infiltration of $p M O G 800:: V e 1$ and $p F A S T::$ Ave 1 may be caused by PTGS. In an attempt to overcome this, Vel and
Avel were coexpressed with silencing suppressor $p 19$ from Tomato bushy stunt virus (Voinnet et al. 2003) or with silencing suppressor $2 b$ from Cucumber mosaic virus (Wang et al. 2004). Unfortunately, expression of $p 19$ induced necrosis in $N$. tabacum by itself, while expression of $2 b$ did not enhance necrosis induced by coexpression of Avel and Vel (Fig. 1A).

To bypass the weak necrosis in $N$. tabacum, we attempted agroinfiltration of pMOG800::Ve1 and pFAST::Ave1 together with silencing suppressor $p 19$ (1:1:1 ratio) in leaves of $N$. benthamiana. Surprisingly, however, coexpression of Avel and $V e 1$ did not result in any HR in N. benthamiana, even up to 14 dpi, while coexpression of tomato $C f-9$ with $C$. fulvum Avr9 in a similar fashion induced clear HR within 4 dpi (Supplementary Fig. 1A).

\section{Various Gateway-compatible binary vectors improve agroinfiltration efficiency.}

Because $N$. benthamiana did not develop necrosis upon coexpression of $\mathrm{Vel}$ and Avel and the silencing suppressors $p 19$ and $2 b$ could not improve the results of the agroinfiltration in $N$. tabacum, we tested various overexpression vectors to improve our results. To this end, the coding sequences of $V$. dahliae Avel and tomato Vel were cloned into various expression vectors that carry the CaMV $35 \mathrm{~S}$ promoter for transgene expression. All constructs were transformed into A. tumefaciens GV3101, and a 1:1 mixture of Agrobacterium carrying 35S::Ve1 and 35S::Ave1 was infiltrated into N. tabacum plants. Leaves were examined for necrosis at 5 dpi (Fig. 2). Interestingly, various expression vectors strongly affected necrosis development, as expression vectors pMOG800, pSol2092, and pEarleyGate100 promoted strong necrosis in which the entire infiltrated area became necrotic (Fig. 2).

Based on these results obtained in N. tabacum, we revisited the agroinfiltration in $N$. benthamiana and infiltrated leaves with a 1:1:1 mixture of Agrobacterium cultures carrying pMOG800::Ve1, pSol2092::Ave1, and silencing suppressor p19. Nevertheless, with these vectors, also, no necrosis was obtained in this species.

\section{C terminally tagged Ve1 is functional in N. tabacum.}

Protein tagging with the green fluorescent protein (GFP) is frequently used for protein localization studies and also for affinity purification (Heese et al. 2007). We cloned the Vel coding sequence without stop codon into various Gatewaycompatible binary vectors that establish a C-terminal fusion to GFP. Only the construct in pSol2095 developed a strong HR

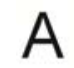

\section{silencing suppressor}

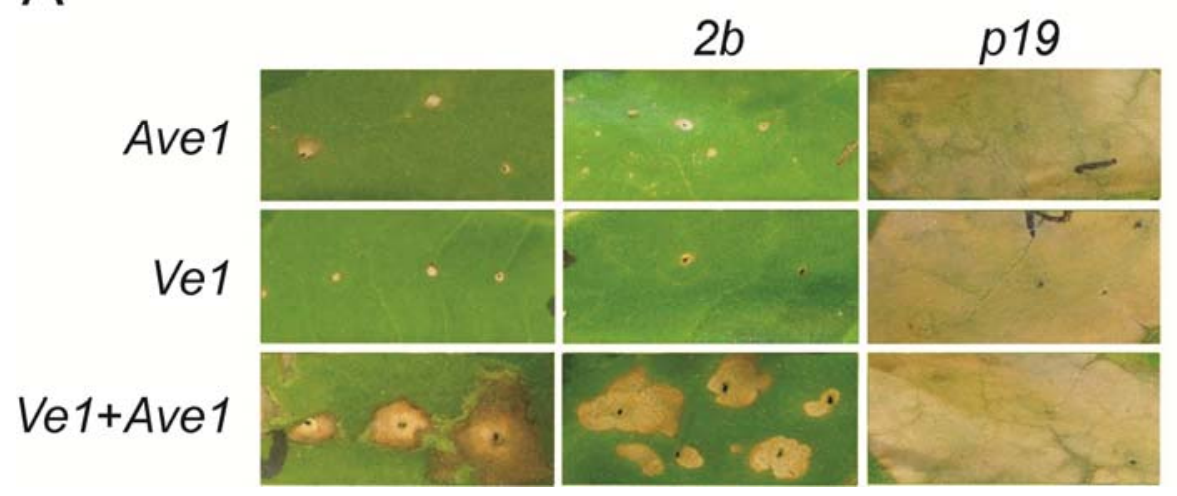

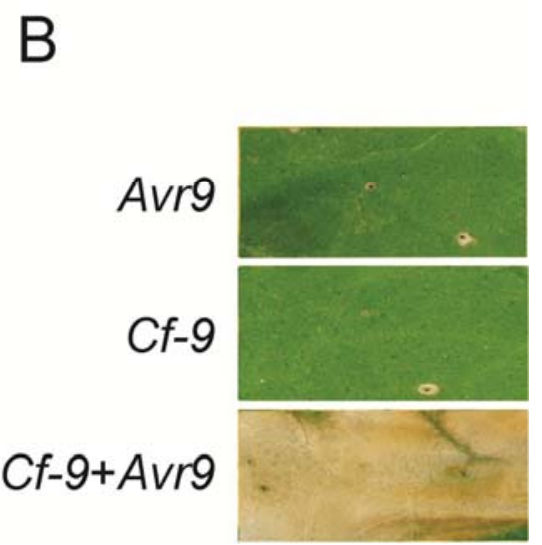

Fig. 1. Coexpression of tomato Vel and Verticillium dahliae Avel induces a hypersensitive response in Nicotiana tabacum. A, Co-agroinfiltration of constructs for constitutive expression of tomato immune receptor gene Vel and the corresponding $V$. dahliae avirulence gene Avel results in necrosis in Nicotiana tabacum at the injection sites. Whereas co-agroinfiltration of silencing suppressor P19 induces necrosis under all conditions, silencing suppressor $2 b$ has no visible effect. B, Coexpression of avirulence gene Avr 9 from the tomato leaf mold fungus Cladosporium fulvum and the corresponding tomato receptor gene $C f-9$ results in strong necrosis in the absence of a silencing suppressor. All photos were taken at 5 days postinfiltration. 
that was comparable to the strong HR that can be obtained with untagged Ve1 (Supplementary Fig. 2).

\section{HR induced by coexpression of $\mathrm{Vel}$ and $A v e 1$}

is restricted to $N$. tabacum and $N$. glutinosa.

Because $N$. tabacum and $N$. benthamiana responded differentially to coexpression of Vel and Avel, we screened additional tobacco genotypes for their response to coexpression of this gene pair. In these assays, agroinfiltration of Vel-GFP served as a marker for efficiency of agroinfiltration, while coexpression of the $C f-9 / A v r 9$ gene pair was used as a positive control for the HR. The results of the agroinfiltration are shown in Table 1. Transient coexpression of the $C f-9 / A v r 9$ gene pair resulted in a hypersensitive response in all of the Nicotiana species tested. In contrast, coexpression of the Vel/Avel gene pair did not result in specific necrosis in most Nicotiana species, although significant

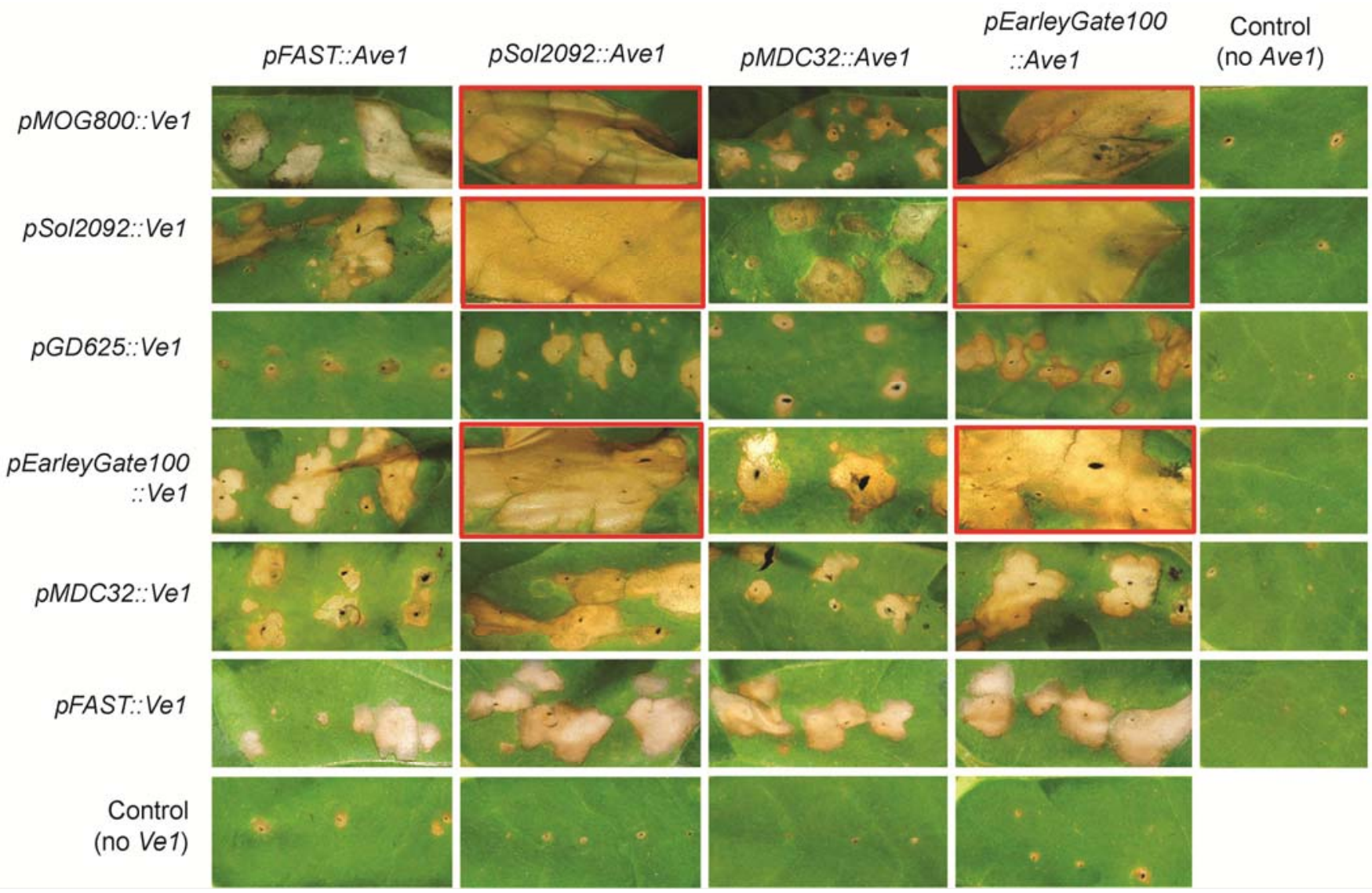

Fig. 2. Comparison of necrosis induced by coexpression of tomato Ve1 and Verticillium dahliae Ave1 in Nicotiana tabacum with various binary vectors. Strongest necrosis is observed upon coexpression of Vel and Ave1 using the pMOG800, pSol2092, and pEarleyGate100 vectors (red outline). All pictures were taken at 5 days postinfiltration.

Table 1. The hypersensitive response (HR) upon coexpression of Vel and Ave1 in various tobacco species

\begin{tabular}{|c|c|c|c|c|c|c|}
\hline Species & 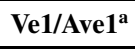 & Cf-9/Avr9 $^{a}$ & Ave $^{\text {a }}$ & GFP $^{\mathbf{b}}$ & PDS $^{\mathbf{c}}$ & Viral symptoms $^{\mathrm{d}}$ \\
\hline N. benthamiana & - & +++ & - & + & +++ & + \\
\hline N. tabacum cv. SR1 & +++ & +++ & - & + & + & ++ \\
\hline N. tabacum cv. White Burley & +++ & +++ & - & + & + & +++ \\
\hline N. tabacum cv. Xanthi & +++ & +++ & - & + & + & + \\
\hline N. tabacum cv. Havana2000 & +++ & +++ & - & + & + & ++ \\
\hline N. tabacum cv. 2.1.1 & +++ & +++ & - & + & + & ++ \\
\hline N. tabacum cv. Samsun & +++ & +++ & - & + & +++ & ++ \\
\hline N. tabacum cv. Samsun NN & ++ & +++ & - & + & +++ & ++ \\
\hline N. rustica & - & ++ & - & + & - & + \\
\hline N. clevelandii & - & ++ & - & + & n.d. & n.d. \\
\hline N. sylvestris & - & +++ & - & + & ++ & ++ \\
\hline N. glutinosa & +++ & +++ & ++ & + & - & ++ \\
\hline N. debneyi & - & +++ & - & + & +++ & ++ \\
\hline N. paniculata & - & ++ & - & n.d. & n.d. & n.d. \\
\hline N. excelsior & - & ++ & - & n.d. & +++ & ++ \\
\hline
\end{tabular}

${ }^{\text {a }}$ HR was monitored at 7 days postinfiltration (dpi). +++= strong HR over the entire infiltrated area; ++= moderate HR; += weak necrosis

${ }^{\mathrm{b}}$ Green flourescent protein (GFP) fluorescence derived from GFP-tagged Ve1 was examined with a UV microscope at 2 dpi. $+=$ clear GFP is visible; n.d.= not determined.

${ }^{\mathrm{c}}$ Degree of photobleaching upon phytoene desaturase (PDS) gene silencing through virus-induced gene silencing. $+++=$ strong photobleaching with the entire leaf turning white; $++=$ moderate photobleaching with more than $50 \%$ of leaf area turning white; $+=$ weak photobleaching of leaf; $-=$ no photobleaching was observed; n.d.= not determined.

d Viral symptoms upon agroinfiltration of $T R V:: P D S .+++=$ strong viral symptoms; ++= moderate viral symptoms; += weak viral symptoms. 
GFP fluorescence was observed upon expression of Ve1-GFP, confirming that the agroinfiltration worked. The Ve1/Ave1induced HR could only be observed in $N$. tabacum cultivars and in Nicotiana glutinosa (Table 1).

\section{N. glutinosa contains a functional Ve1 ortholog.}

Intriguingly, agroinfiltration of only Avel into Nicotiana glutinosa leaves already induced HR, albeit not as strong as the HR induced by coexpression of Avel with Vel, while agroinfiltration of $\mathrm{Vel}$ alone did not induce HR (Table 1; Fig. 3A). This suggests that $N$. glutinosa contains a functional Vel ortholog. To confirm the presence of a functional Ve1 ortholog in $N$. glutinosa, we tested the resistance of $N$. glutinosa to race 1 $V$. dahliae by inoculating 3-week-old $N$. glutinosa plants with race $1 V$. dahliae JR2. At 2 weeks postinoculation, it was clearly observed that the inoculated $N$. glutinosa plants were resistant to the $V$. dahliae infection, as plants were completely devoid of wilt disease symptoms (Fig. 3B). Importantly, when inoculated with an Avel deletion strain (de Jonge et al. 2012), the $N$. glutinosa plants displayed clear symptoms of Verticillium wilt disease, including stunting, wilting, cholorosis, and necrosis at 14 days postinoculation (Fig. 3B). We subsequently attempted to clone the Vel homolog from $N$. glutinosa, making use of primers designed on the tomato $\mathrm{Vel}$ sequence. Although cloning of complete coding sequence failed so far, a 461-bp product was amplified from $N$. glutinosa cDNA showing a high degree of homology to tomato Vel (Supplementary Fig. 4). Collectively, these experiments demonstrate that $N$. glutinosa contains a functional $\mathrm{Ve} 1$ ortholog that provides resistance against race $1 \mathrm{~V}$. dahliae through recognition of Ave1.

\section{Tobacco rattle virus (TRV)-based VIGS in $N$. tabacum.}

In an attempt to establish VIGS in N. tabacum, a 1:1 mixture of A. tumefaciens cultures carrying $p T R V 1$ and $p T R V 2:: P D S$ to target the phytoene desaturase (PDS) gene was infiltrated into two cotyledons of five 2- to 3-week-old plants of the N. tabacum cultivar SR1. Photobleaching symptoms were observed in all of the five agroinfiltrated $N$. tabacum cv. SR1 plants at 3 weeks postinfiltration. However, $P D S$ silencing was only consistently observed in the stems of the plants, as these were fully bleached

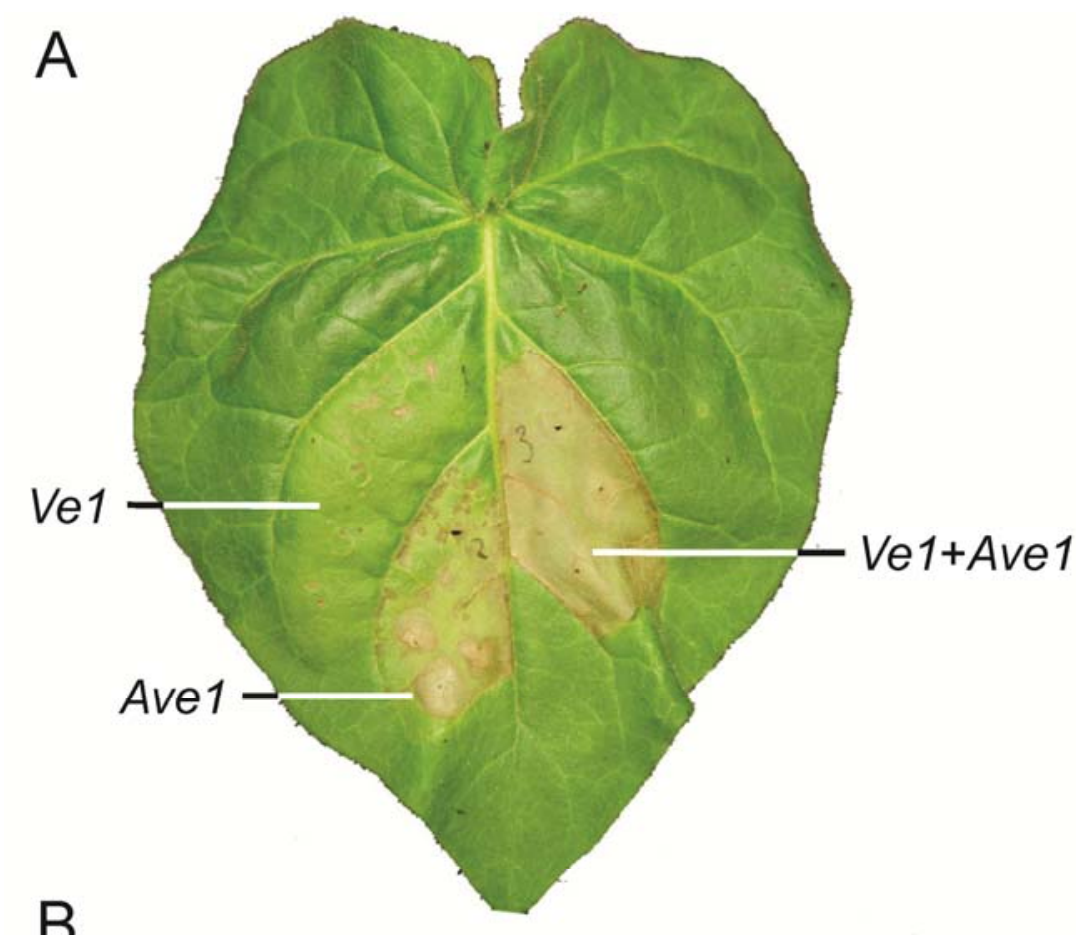

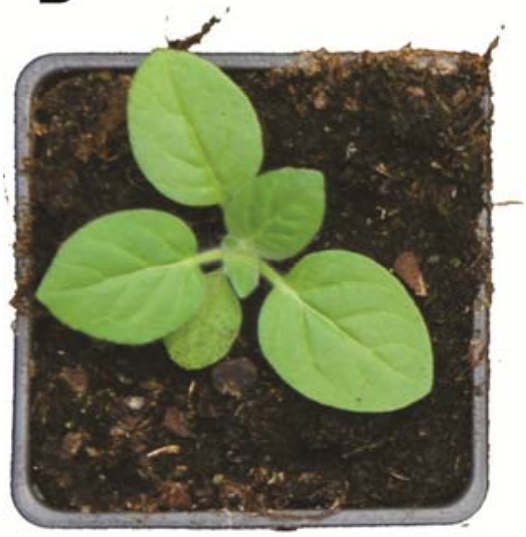

mock

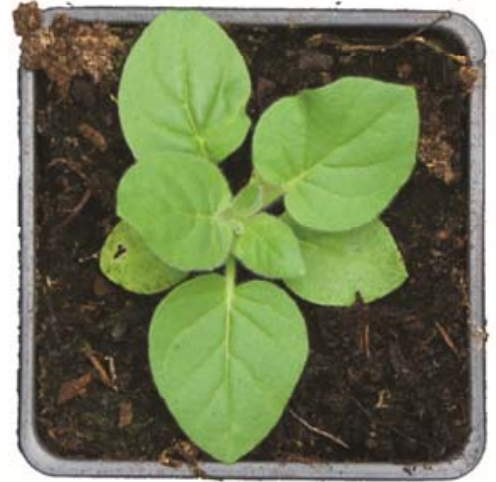

race $1 \mathrm{~V}$. dahliae

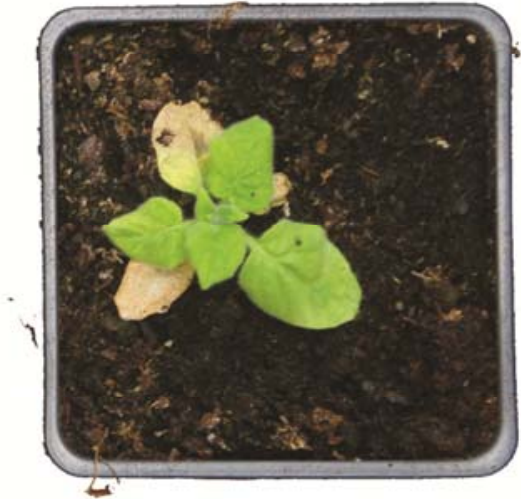

$\triangle$ Ave1

Fig. 3. Nicotiana glutinosa contains a functional Ve1 ortholog. A, Agroinfiltration of Ave1, Ve1, and a combination thereof in N. glutinosa. In contrast to agroinfiltration of $\mathrm{Ve} 1$ alone, agroinfiltration of Ave1 alone in $N$. glutinosa induces a hypersensitive response. The picture was taken at 7 days postinfiltration. B, $N$. glutinosa is resistant against race $1 \mathrm{~V}$. dahliae but not against an Ave 1 deletion strain $(\Delta$ Ave 1$)$. Photos were taken at 14 days postinoculation. 
(Supplementary Fig. 3). In the leaves, photobleaching was found to be restricted to a limited area immediately adjacent to some of the veins, suggesting that the TRV-based VIGS is not efficient in N. tabacum under the conditions used.

Next, we tested in which of the tobacco species TRV could induce efficient gene silencing. The degree of photobleaching varied among the species, from no bleaching in $N$. rustica and $N$. glutinosa to very strong bleaching in $N$. benthamiana, $N$. debneyi, and N. excelsior. Interestingly, while in most N. tabacum cultivars, photobleaching occurred only weakly or was limited in leaves (Table 1), highly efficient photobleaching was observed in leaves of the cultivars Samsun and Samsun NN (Table 1; Fig. 4). As it has been reported that the EDS1 (enhanced disease susceptibility 1) gene is required for Vel-mediated Verticillium resistance in tomato and Arabidopsis (Fradin et al. 2009; 2011; Hu et al. 2005), the role of this gene in the HR upon coexpression of $\mathrm{Vel}$ and Avel was assessed. At 3 weeks after TRV infection, TRV-inoculated plants were smaller than noninoculated plants. Coexpression of $\mathrm{Vel}$ and Avel in N. tabacum cv. Samsun plants inoculated with recombinant virus to target GFP expression as a control ( $p T R V: G F P)$ resulted in a clear HR within 2 days (Fig. 5A). As expected however, $N$. tabacum cv. Samsun plants inoculated with recombinant virus to target EDS1 expression ( $p T R V: E D S 1$ ) showed a significantly compromised HR upon coexpression of Vel and Ave1, confirming the involvement of EDS1 in Ve1mediated resistance signaling (Fig. 5A). We also targeted expression of $\mathrm{NRCl}$ (a nucleotide binding-LRR protein required for HR-associated cell death 1), which has similarly been reported to be involved in Ve1-mediated resistance in tomato (Fradin et al. 2009). However, similar to SGT1, inoculation of $N$. tabacum cv. Samsun with $p T R V: N R C 1$ strongly affected plant development, and plants could not be used for coexpression of Vel and Avel (Fig. 5B).

\section{DISCUSSION}

Coexpression of Ve1 and Ave1 induces HR only in particular Nicotiana species.

In this study, we investigated the occurrence of HR in leaves of various Nicotiana species upon coexpression of tomato Vel and $V$. dahliae Ave1, revealing that HR can only be obtained in
$N$. tabacum and $N$. glutinosa. Intriguingly, HR was not observed in the widely used tobacco model species $N$. benthamiana. This is in contrast to the HR that is induced by the tomato Cf- 4 and Cf-9 immune receptors that belong to the same RLP family as Ve1 and that provide resistance against strains of the foliar pathogen C. fulvum and that carry Avr4 and Avr9, respectively, which can be induced upon coexpression with these corresponding effector genes (Table 1) (Van der Hoorn et al. 2000). This suggests that most tobacco species lack at least one important signal transduction component that is required for Vel-mediated immunity against $V$. dahliae and further confirms the earlier finding that $\mathrm{Cf}$ and $\mathrm{Ve} 1$ signaling is distinct, despite the fact that both immune receptors belong to the same RLP family (Fradin et al. 2009, 2011; Vossen et al. 2010). The failure of $\mathrm{Ve} 1$ to mediate $\mathrm{HR}$ in most tobacco species was rather unexpected, considering that Ve1 orthologs that have been implicated in resistance against the broad host range pathogen V. dahliae are more widespread than $\mathrm{Cf}$ orthologs, which are confined to tomato as the only known host of $C$. fulvum (Fradin et al. 2009; Thomma et al. 2005, 2011). This finding is even more surprising considering that a functional Vel ortholog was identified even within the Nicotiana family, in $N$. glutinosa and that heterologous expression of tomato Vel in Arabidopsis results in resistance against race 1 Verticillium strains.

\section{Agroinfiltration as a method to identify functional Vel orthologs.}

Agroinfiltration has been demonstrated to be effective for transient expression in many plant species, including tobacco, lettuce, Arabidopsis, radish, pea, lupine, flax, tomato, grapevine, and switchgrass (Santos-Rosa et al. 2008; van der Gheynst et al. 2008; Van der Hoorn et al. 2000; Wroblewski et al. 2005). This allows the use of agroinfiltration to identify candidate functional Vel orthologous genes by coexpression with Avel. Since $V$. dahliae is an economically important pathogen, many efforts have been made to identify Vel orthologs from various plant species, within and outside the Solanaceae family, including SlVel cloned from Solanum lycopersicoides (Chai et al. 2003), StVel cloned from Solanum tuberosum (Simko et al. 2004), StVe cloned from Solanum torvum Swartz (Fei et al. 2004), $m$ Vel cloned from Mentha longifolia (Vining and Davis
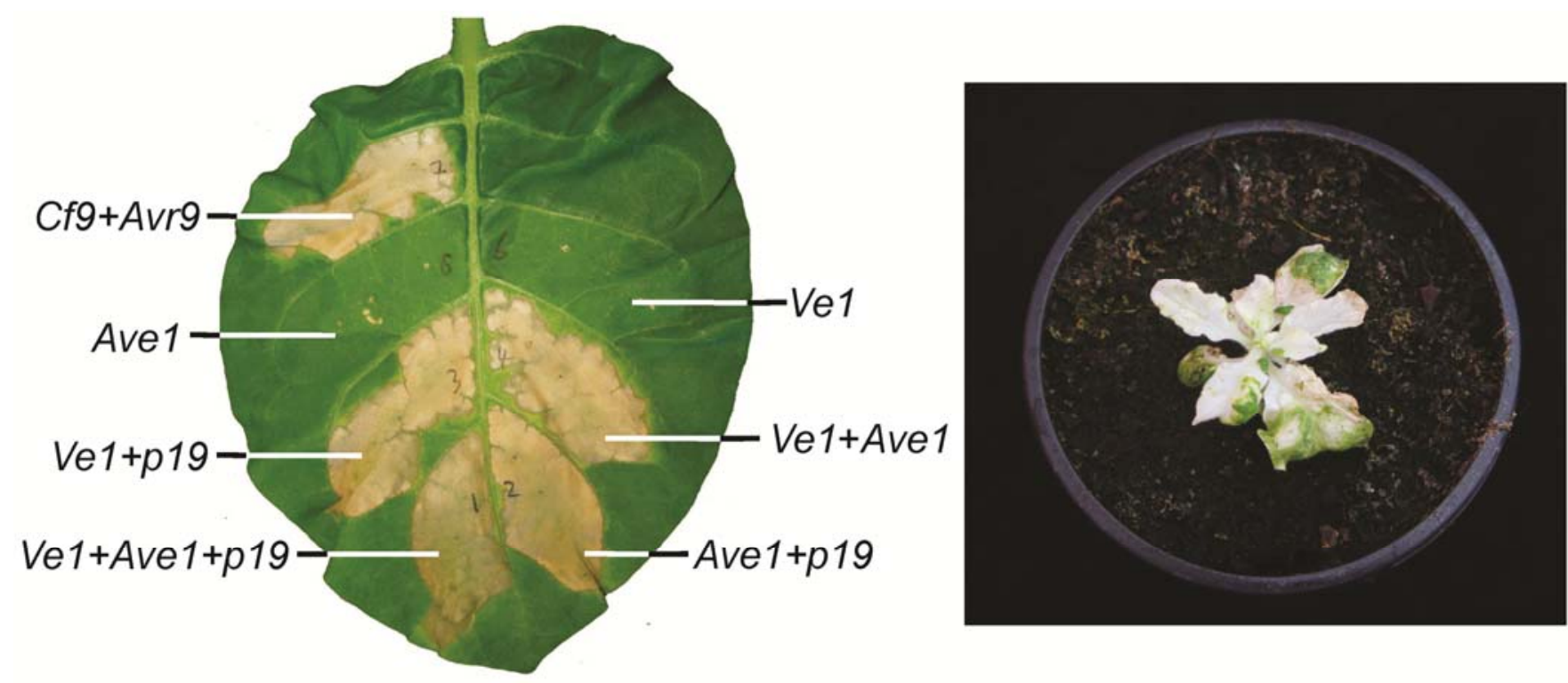

Fig. 4. Agroinfiltration and virus-induced gene silencing (VIGS) in Nicotiana tabacum cv. Samsun. On the left, co-infiltration of Ve1 and Ave1 induced a hypersensitive response in N. tabacum cv. Samsun. On the right, VIGS of the phytoene desaturase (PDS) gene leads to photobleaching in leaves of . tabacum cv. Samsun. 
2009; Vining et al. 2007), GbVe cloned from Gossypium barbadense (Zhang et al. 2011), and the StoVel gene cloned from Solanum torvum (Liu et al. 2012). Functionality of the putative Vel orthologs was assessed by subsequent transformation into susceptible plants followed by Verticillium inoculation, which, depending on the plant species, is laborious (Fradin et al. 2009; Kawchuk et al. 2001; Liu et al. 2012; Vining and Davis 2009). We have previously shown that transfer of tomato Vel to Arabidopsis resulted in resistance against race 1 Verticillium strains (Fradin et al. 2011), providing a relatively fast method to assess functionality of Ve1 orthologs (Zhang et al. 2011). Nevertheless, the engineering of stable Arabidopsis transgenes still requires a couple of months and much work to get homo- zygous transgenic Arabidopsis with those Ve orthologs. Our results indicate that coexpression of such a candidate with Avel in N. tabacum may be a much more rapid (matter of days) method to identify functional $\mathrm{Vel}$ orthologs.

\section{Expression vectors differentially affect \\ Ave1/Ve1-induced necrosis in tobacco.}

Our results show that coexpression of the secreted Verticillium effector Avel and the corresponding tomato immune receptor Vel by agroinfiltration induces HR in N. tabacum but not in $N$. benthamiana. Since silencing suppressors could not improve the strength of the HR in $N$. tabacum, we tested various Gateway-compatible binary vectors that mediate transgene

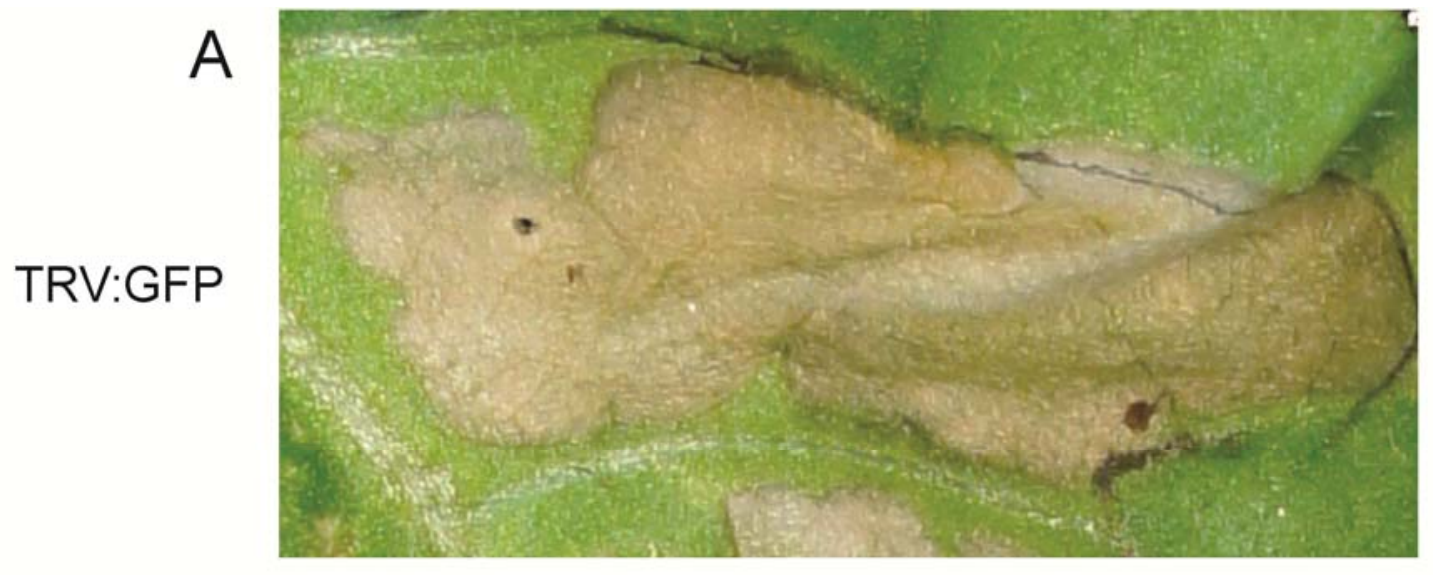

TRV:EDS1

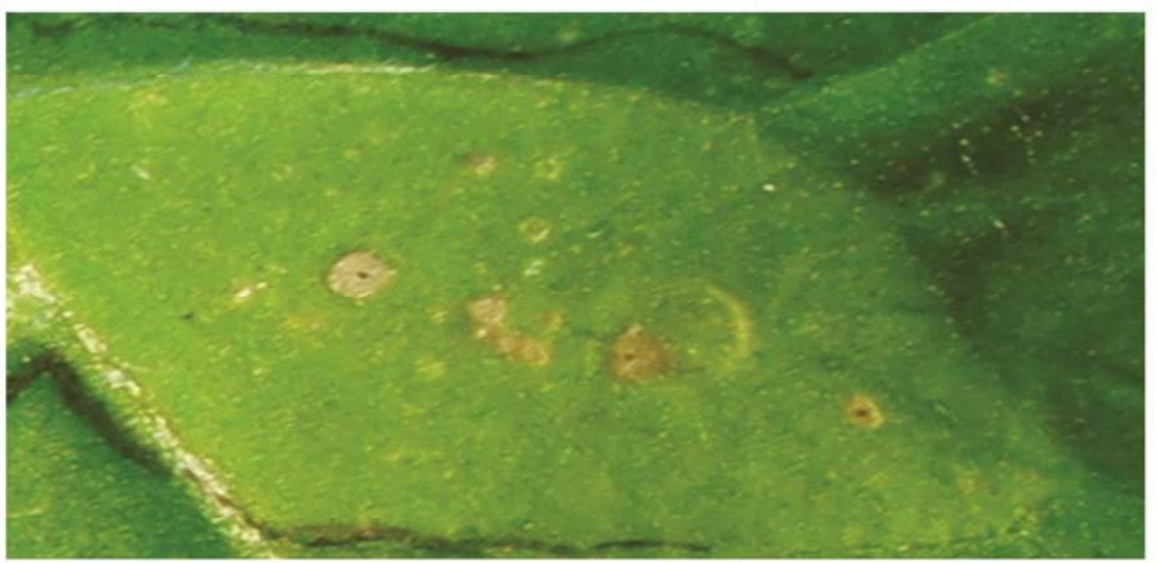

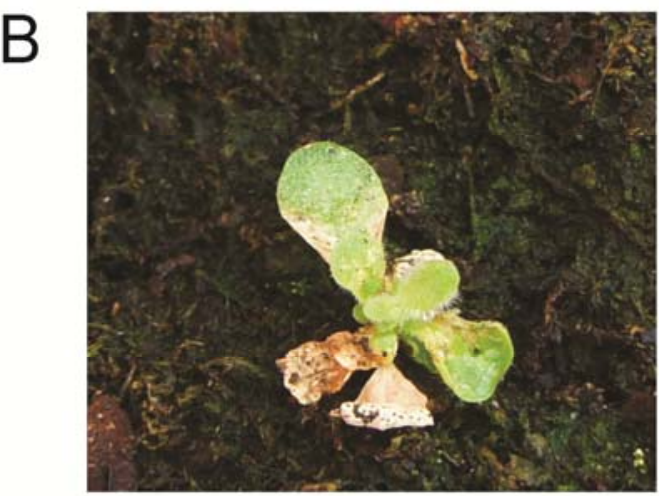

TRV:NRC1

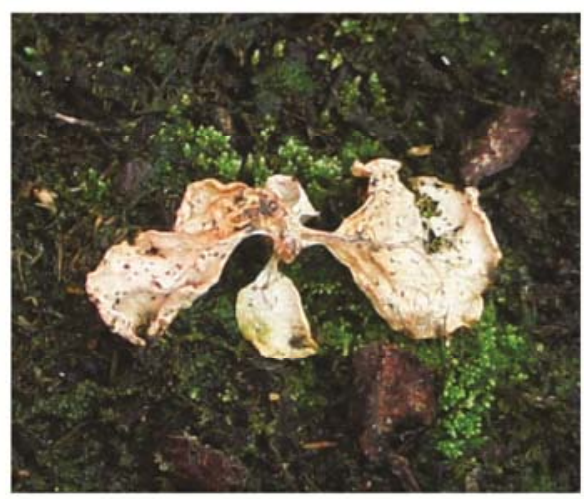

TRV:SGT1

Fig. 5. Coexpression of Ve1 and Ave1 in Nicotiana tabacum cv. Samsun after virus-induced gene silencing. A, N. tabacum cv. Samsun plants were inoculated with a recombinant Tobacco rattle virus (TRV) targeting a green fluorescent protein gene as a control (TRV:GFP) or recombinant TRV targeting the EDS1 gene (TRV:EDS1). At 5 days postinfiltration, compromised hyypersensitive response was observed in EDS1-silenced plants. B, TRV:NRC1-inoculated $N$. tabacum cv. Samsun plants show a strong developmental phenotype while $S G T 1$-silencing results in lethality. 
expression driven by the CaMV $35 \mathrm{~S}$ promoter. These vectors differentially affected the level of necrosis induced by Ve1mediated recognition of Ave 1 in tobacco. These differences may be caused by different transformation efficiencies of the various vectors or by different expression rates of these vectors. Whereas recombinant protein could not be detected in Western analysis upon agroinfiltration of $p K 7 F W G 2:: V e 1:: G F P$, which only triggers weak HR, ample recombinant protein was detected in Western analysis upon agroinfiltration of pSol2095:: Vel::GFP, which activates strong HR. However, as it was our aim to set up an appropriate expression system for Ve1/Aveinduced HR, we did not further invest to discover the technical reasons for differences in vector efficiencies.

\section{TRV-based VIGS for functional analysis in N. tabacum.}

Previously, by using TRV-based VIGS in tomato, we established a method for functional analysis of genes that are involved in Verticillium defense (Fradin et al. 2009). Several candidate genes were silenced in tomato, followed by inoculation with race $1 \mathrm{~V}$. dahliae revealing a role in Ve1-mediated Verticillium defense for some of them (Fradin et al. 2009, 2011; Vossen et al. 2010). As TRV induces only mild viral symptoms and infects large areas of adjacent cells, TRV-mediated VIGS has been used extensively in $N$. benthamiana and tomato, and consequently, many TRV-based VIGS vectors targeting potential defense components are available (Chen et al. 2009; Fradin et al. 2009; Gabriëls et al. 2006, 2007; Ho et al. 2009; Stulemeijer et al. 2007; Vossen et al. 2010). Since tomato and tobacco are close relatives, they share a high degree of coding sequence homology between orthologs, allowing TRV constructs targeting tomato to be successfully used in tobacco and vice versa (Fradin et al. 2009; Gabriëls et al. 2006; Liebrand et al. 2012; Senthil-Kumar et al. 2007; Velasquez et al. 2009).

Recently, the development of VIGS in Nicotiana tabacum using Tobacco curly shoot virus was described (Huang et al. 2011). However, many TRV-based VIGS vectors targeting potential defense components are available. Therefore, we tried to establish TRV-based VIGS in combination with agroinfiltration in tobacco, to study Ve1-signaling. And although TRV-based VIGS has been reported in N. tabacum (Ryu et al. 2004), we found it did not induce sufficient gene silencing in leaves of $N$. tabacum cv. SR1, which we used for HR induced by coexpression of Vel and Avel. The screening of Nicotiana species demonstrated that $N$. tabacum cv. Samsun is able to develop an HR upon coexpression of $\mathrm{Vel}$ and Avel and is amenable to TRVbased VIGS. By the silencing of EDS1, which is known to be required for Ve1-mediated resistance in tomato and Arabidopsis (Fradin et al. 2009, 2011; Hu et al. 2005), we demonstrated that ATTA in combination with VIGS can be employed to unravel Vel-mediated defense signaling using tobacco.

\section{MATERIALS AND METHODS}

\section{Plant materials.}

Tobacco plants were grown in soil in the greenhouse with the following settings: $16-\mathrm{h}, 21^{\circ} \mathrm{C}$ day and $8-\mathrm{h}, 19^{\circ} \mathrm{C}$ night periods, with $70 \%$ relative humidity, and $100 \mathrm{~W} \cdot \mathrm{m}^{-2}$ supplemental light when the light intensity dropped below $150 \mathrm{~W} \cdot \mathrm{m}^{-2}$.

\section{Expression vectors pSol2092 and pSol2095.}

To construct pSol2092 and pSol2095, first a backbone was constructed that contains the NPTIII, trfA, oriV, T-DNA left border and part of the nptII plant selection marker from pEAQHT (Sainsbury et al. 2009), the aadA gene and the pBR322 origin of replication from pPZP200 (Hajdukiewicz et al. 1994), the T-DNA right border, and part of the nptII plant selection marker derived from pMOG800 (Knoester et al. 1998).
Subsequently, the expression cassettes from pB7WG2 and pK7FWG2 (Karimi et al. 2002) were inserted in between the right border of the T-DNA region and the NPTII plant selection marker to create pSol2092 and pSol2095, respectively. During the process, a number of restriction sites were added and removed. DNA fragments that were created by polymerase chain reaction (PCR) were sequence-verified.

\section{Generation of constructs}

for over-expression of $\mathrm{VeI}$ and Ave1.

The coding sequence of $V$. dahliae Avel (de Jonge et al. 2012) was PCR-amplified using primers containing AttB1 and AttB2 sites for Gateway-compatible cloning: Vd-F, 5'-GG GGACAAGTTTGTACAAAAAAGCAGGCTATGAAGCTTT CTACGCTT-3' and Vd-R, 5'-GGGGACCACTTTGTACAAG AAAGCTGGGTTTATATCTGTCTAAATTC-3'. The resulting PCR product was cleaned from $1 \%$ agarose gel, using the QIAquick gel extraction kit (Qiagen, Valencia, CA, U.S.A.), and was transferred into donor vector pDONR207, using Gateway BP Clonase II enzyme mix (Invitrogen, Carlsbad, CA, U.S.A.) to generate entry vector pDONR207::Ave1. Similarly, the tomato Ve1 coding sequence was PCR-amplified from pMOG800::Ve1 (Fradin et al. 2009), using primers attBVe1-F，5'-GGGGACAAGTTTGTACAAAAAAGCAGGCTAT GAAAATGATGGCAACTCT-3' and attB-Ve1-R 5'-GGGGAC CACTTTGTACAAGAAAGCTGGGTAGTCTTTCTTGAAA ACCAAA -3'. The PCR product was cleaned from agarose gel and was transferred into Gateway donor vector pDONR207 to generate entry vector pDONR207::Ve1. The entry constructs pDONR207::Ave1 and pDONR207::Ve1 were subsequently cloned into Gateway destination vector pFAST_R02 (Shimada et al. 2010), pMDC32 (Curtis and Grossniklaus 2003), pEarleyGate100 (Earley et al. 2006), pGD625 (Dekkers et al. 2008), and pSol2092, using Gateway LR Clonase II enzyme mix (Invitrogen) to generate expression constructs driven by the CaMV 35S promoter. The expression constructs were transformed into Escherichia coli and transformants were checked by colony-PCR analysis, using primers AttB1F 5'ACAAGTTTGTACAAAAAAGCAGGCT-3' and AttB2R 5' ACCACTTTGTACAAGAAAGCTGGGT-3'. The expression constructs were subsequently transformed into A. tumefaciens GV3101 (Hellens et al. 2000) by electroporation. Finally, the plasmids were isolated from A. tumefaciens transformants and were sequenced.

\section{Generation of constructs for Ve1-GFP fusion.}

To generate Ve1 fused at the $3^{\prime}$ end to a GFP tag, the Vel coding sequence lacking the stop codon was PCR-amplified using primers containing Gateway attB sites: attB-Ve1-F, 5'GGGGACAAGTTTGTACAAAAAAGCAGGCTATGAAAAT GATGGCAACTCT-3' and attB-Ve1-R-SC, 5'-GGGGACCA CTTTGTACAAGAAAGCTGGGTACTTTCTTGAAAACCA AAG-3'. The PCR fragment was cloned into pDONR207 (Invitrogen) through a Gateway $\mathrm{BP}$ reaction to generate entry vector pDONR207::Ve1-SC. Subsequently, pDONR207::Ve1SC was transferred into the Gateway-compatible destination vectors pK7FWG2 (Karimi et al. 2002), pMDC83 (Curtis et al. 2003), pGWB451 (Nakagawa et al. 2007), and pSol2095 to generate expression constructs driven by the constitutive CaMV 35S promoter and C-terminally tagged to GFP. Fusion constructs were transformed into A. tumefaciens GV3101 by electroporation.

\section{A. tumefaciens-mediated transient expression.}

A. tumefaciens containing expression constructs was infiltrated into tobacco plants as described previously (Van der Hoorn et al. 2000). Briefly, an overnight culture of A. tumefa- 
ciens cells was harvested at an optical density at $600 \mathrm{~nm}$ $\left(\mathrm{OD}_{600}\right)$ of 0.8 to 1 by centrifugation and was resuspended to a final $\mathrm{OD}_{600}$ of 2. A. tumefaciens cultures containing constructs to express Ave 1 and $V e$ proteins were mixed in a 1:1 ratio and were infiltrated into leaves of 5- to 6-week-old tobacco plants. After agroinfiltration, plants were grown in the climate room at $22^{\circ} \mathrm{C}$ and $19^{\circ} \mathrm{C}$ during 16 -h day and 8-h night periods, respectively, with $70 \%$ relative humidity. At 5 dpi, leaves were examined for necrosis.

\section{Amplification of a Vel homolog from $N$. glutinosa.}

Total RNA of $N$. glutinosa seedlings was extracted using the QIAGEN RNeasy extraction kit (Qiagen). First-strand cDNA was synthesized from $1 \mu \mathrm{g}$ of total RNA, using the SuperScript III cDNA synthesis kit (Invitrogen) according to the manufacturers' instructions. The partial coding sequence of N. glutinosa was PCR-amplified from cDNA with $P f u$ highfidelity enzyme (Promega, Madison, WI, U.S.A.), using primer Ve1-F4 (5'-GGTCTCACCCACCTGAATCTTTC-3') and Ve1R5 (5'-CCTTGTAAGTTATTCGCACTGA-3'). PCR was performed for 30 cycles, with denaturing at $95^{\circ} \mathrm{C}$ for $30 \mathrm{~s}$, annealing at $55^{\circ} \mathrm{C}$ for $30 \mathrm{~s}$, and elongation at $72^{\circ} \mathrm{C}$ for $60 \mathrm{~s}$. The resulting 461-bp PCR product was cleaned from $1 \%$ agarose gel, was cloned into the pGEM-T Easy vector (Promega), and was sequenced using the M13F primer (5'-TGTAAAACGAC GGCCAGT-3').

\section{VIGS.}

The TRV2 constructs TRV:GFP, TRV:PDS, TRV:EDS1, TRV: $S G T 1$, and TRV:NRC1 have been described before (Gabriëls et al. 2006). To establish VIGS in tobacco, cotyledons of 2- to 3week-old tobacco seedlings were infiltrated with 1:1 mixtures of pTRV1 and pTRV2 constructs, using a needleless 1-ml syringe as described by Van der Hoorn and associates (2000). Leaves were superficially wounded with a needle to improve infiltration. Photobleaching was observed at 3 weeks postinfiltration upon inoculation of $T R V: P D S$.

\section{Verticillium inoculations.}

Race $1 \mathrm{~V}$. dahliae JR2 and the Ave1 deletion strain ( $\Delta$ Ave1) (de Jonge et al. 2012) were grown on potato dextrose agar at $22^{\circ} \mathrm{C}$. V. dahliae conidia were harvested from 7- to 14-day-old fungal plates and were washed with tap water. The conidia were suspended to a final concentration of $10^{6}$ conidia $/ \mathrm{ml}$. For inoculation, 3-week-old $N$. glutinosa plants were gently uprooted and the roots were rinsed in tap water. The roots were then dipped in the conidial suspension for $3 \mathrm{~min}$. As a control, plants were mock-inoculated in tap water. After mock-inoculation, plants were transferred to soil. The inoculated plants were evaluated by observing disease symptoms such as stunting of plant growth, wilting, chlorosis, and necrosis at $14 \mathrm{dpi}$.

\section{ACKNOWLEDGMENTS}

B. P. H. J. Thomma and H. P. van Esse are supported by a Vidi and a Veni grant, respectively, of the Netherlands Organization for Scientific Research (NWO-ALW), and Z. Zhang is supported by a sandwich fellowship of the Wageningen University. This research was furthermore supported by the Centre for BioSystems Genomics (CBSG) and ERA-NET Plant Genomics. We acknowledge W. Tameling, G. Wang, J. Du, C. Huang and X. Zhou for their generous gifts of materials and valuable discussions. We furthermore thank B. Essenstam and H. Smid for excellent plant care.

\section{LITERATURE CITED}

Armen, J., and Shoemaker, P. B. 1985. Histopathology of resistant and susceptible tomato cultivars inoculated with Verticillium dahliae Kleb race 1 and race 2 . Phytopathology 75:1361-1362.
Bar, M., and Avni, A. 2009. EHD2 inhibits ligand-induced endocytosis and signaling of the leucine-rich repeat receptor-like protein LeEix2. Plant J. 59:600-611.

Chai, Y. R., Zhao, L. X., Liao, Z. H., Sun, X. F., Zuo, K. J., Zhang, L., Wang, S. G., and Tang, K. X. 2003. Molecular cloning of a potential Verticillium dahliae resistance gene SlVe1 with multi-site polyadenylation from Solanum licopersicoides. DNA Seq. 14:375-384.

Chapman, S., Kavanagh, T., and Baulcombe, D. 1992. Potato virus X as a vector for gene expression in plants. Plant J. 2:549-557.

Chen, Y. Y., Lin, Y. M., Chao, T. C., Wang, J. F., Liu, A. C., Ho, F. I., and Cheng, C. P. 2009. Virus-induced gene silencing reveals the involvement of ethylene-, salicylic acid- and mitogen-activated protein kinaserelated defense pathways in the resistance of tomato to bacterial wilt. Physiol. Plant. 136:324-335.

Curtis, M. D., and Grossniklaus, U. 2003. A gateway cloning vector set for high-throughput functional analysis of genes in planta. Plant Physiol. 133:462-469.

de Jonge, R., van Esse, P., Maruthachalam, K., Bolton, M. D., Santhanam, P., Saber, M. K., Zhang, Z., Usami, T., Lievens, B., Subbarao, K. V., and Thomma, B. P. H. J. 2012. Tomato immune receptor Vel recognizes effector of multiple fungal pathogens uncovered by genome and RNA sequencing. Proc. Natl. Acad. Sci. U.S.A. 109:5110-5115.

Dekkers, B. J. W., Schuurmans, J. A. M. J., and Smeekens, S. C. M. 2008. Interaction between sugar and abscisic acid signalling during early seedling development in Arabidopsis. Plant Mol. Biol. 67:151-167.

Earley, K. W., Haag, J. R., Pontes, O., Opper, K., Juehne, T., Song, K. M., and Pikaard, C. S. 2006. Gateway-compatible vectors for plant functional genomics and proteomics. Plant J. 45:616-629.

Faino, L., de Jonge, R., and Thomma, B. 2012. The transcriptome of Verticillium dahliae-infected Nicotiana benthamiana determined by deep RNA sequencing. Plant Signal. Behav. 7:0-1

Fei, J., Chai, Y. R., Wang, J., Lin, J., Sun, X. F., Sun, C., Zuo, K. J., and Tang, K. X. 2004. cDNA cloning and characterization of the Ve homologue gene StVe from Solanum torvum Swartz. DNA Seq. 15:88-95.

Fradin, E. F., and Thomma, B. P. H. J. 2006. Physiology and molecular aspects of Verticillium wilt diseases caused by $V$. dahliae and V. alboatrum. Mol. Plant Pathol. 7:71-86.

Fradin, E. F., Zhang, Z., Ayala, J. C. J., Castroverde, C. D. M., Nazar, R. N., Robb, J., Liu, C. M., and Thomma, B. P. H. J. 2009. Genetic dissection of Verticillium wilt resistance mediated by tomato Ve1. Plant Physiol. 150:320-332.

Fradin, E. F., Abd-El-Haliem, A., Masini, L., van den Berg, G. C., Joosten, M. H. A. J., and Thomma, B. P. H. J. 2011. Interfamily transfer of tomato Vel mediates Verticillium resistance in Arabidopsis. Plant Physiol. 156:2255-2265

Fritz-Laylin, L. K., Krishnamurthy, N., Tor, M., Sjolander, K. V., and Jones, J. D. G. 2005. Phylogenomic analysis of the receptor-like proteins of rice and Arabidopsis. Plant Physiol. 138:611-623.

Gabriëls, S. H. E. J., Takken, F. L. W., Vossen, J. H., de Jong, C. F., Liu, Q., Turk, S. C. H. J., Wachowski, L. K., Peters, J., Witsenboer, H. M. A., de Wit, P. J. G. M., and Joosten, M. H. A. J. 2006. cDNA-AFLP combined with functional analysis reveals novel genes involved in the hypersensitive response. Mol. Plant-Microbe Interact. 19:567-576.

Gabriëls, S. H. E. J., Vossen, J. H., Ekengren, S. K., van Ooijen, G., AbdEl-Haliem, A. M., van den Berg, G. C. M., Rainey, D. Y., Martin, G. B., Takken, F. L. W., de Wit, P. J. G. M., and Joosten, M. H. A. J. 2007. An NB-LRR protein required for HR signalling mediated by both extraand intracellular resistance proteins. Plant J. 50:14-28.

Goodin, M. M., Zaitlin, D., Naidu, R. A., and Lommel, S. A. 2008. Nicotiana benthamiana: Its history and future as a model for plant-pathogen interactions. Mol. Plant-Microbe Interact. 21:1015-1026.

Hajdukiewicz, P., Svab, Z., and Maliga, P. 1994. The small, versatile pPZP family of Agrobacterium binary vectors for plant transformation. Plant Mol Biol. 25:989-994.

Heese, A., Hann, D. R., Gimenez-Ibanez, S., Jones, A. M. E., He, K., Li, J., Schroeder, J. I., Peck, S. C., and Rathjen, J. P. 2007. The receptorlike kinase SERK3/BAK1 is a central regulator of innate immunity in plants. Proc. Natl. Acad. Sci. U.S.A. 104:12217-12222.

Hellens, R. P., Edwards, E. A., Leyland, N. R., Bean, S., and Mullineaux, P. M. 2000. pGreen: A versatile and flexible binary Ti vector for agrobacterium-mediated plant transformation. Plant Mol.Biol. 42:819-832.

Ho, F. I., Chen, Y. Y., Lin, Y. M., Cheng, C. P., and Wang, J. F. 2009. A Tobacco rattle virus-induced gene silencing system for a soil-borne vascular pathogen Ralstonia solanacearum. Bot. Stud. 50:413-424.

Hu, G. S., deHart, A. K. A., Li, Y. S., Ustach, C., Handley, V., Navarre, R., Hwang, C. F., Aegerter, B. J., Williamson, V. M., and Baker, B. 2005. EDS1 in tomato is required for resistance mediated by TIR-class $\mathrm{R}$ genes and the receptor-like R gene Ve. Plant J. 42:376-391.

Huang, C.J., Zhang, T., Li, F.F., Zhang, X.Y., and Zhou, X.P. 2011. Development and application of an efficient virus-induced gene silencing sys- 
tem in Nicotiana tabacum using geminivirus alphasatellite. J. Zhejiang Univ. Sci. B. 12:83-92.

Johansen, L. K., and Carrington, J. C. 2001. Silencing on the spot. Induction and suppression of RNA silencing in the Agrobacterium-mediated transient expression system. Plant Physiol. 126:930-938.

Karimi, M., Inzé, D., and Depicker, A. 2002. GATEWAY ${ }^{\mathrm{TM}}$ vectors for agrobacterium-mediated plant transformation. Trends Plant Sci. 7:193195

Kawchuk, L. M., Hachey, J., Lynch, D. R., Kulcsar, F., van Rooijen, G. Waterer, D. R., Robertson, A., Kokko, E., Byers, R., Howard, R. J., Fischer, R., and Prüfer, D. 2001. Tomato Ve disease resistance genes encode cell surface-like receptors. Proc. Natl. Acad. Sci. U.S.A. 98:6511-6515.

Klosterman, S. J., Atallah, Z. K., Vallad, G. E., and Subbarao, K. V. 2009. Diversity, pathogenicity, and management of Verticillium species. Annu. Rev. Phytopathol. 47:39-62.

Knoester, M., van Loon, L.C., van den Heuvel, J., Hennig, J., Bol, J.F., and Linthorst, H.J. 1998. Ethylene-insensitive tobacco lacks nonhost resistance against soil-borne fungi. Proc. Natl. Acad. Sci. U.S.A. 95:1933-1937.

Liebrand, T. W. H., Smit, P., Abd-El-Haliem, A., de Jonge, R., Cordewener, J. H. G., America, A. H. P., Sklenar, J., Jones, A. M. E., Robatzek, S., Thomma, B. P. H. J., Tameling, W. I. L., Joosten, M. H. A. J. 2012 ER-quality control chaperones facilitate the biogenesis of $\mathrm{Cf}$ receptor-like proteins involved in pathogen resistance of tomato. Plant Physiol. 159:1819-1833.

Liu, S. P., Zhu, Y. P., Xie, C., Jue, D. W., Hong, Y. W., Chen, M., Hubdar, A., and Yang, Q. 2012. Transgenic potato plants expressing StoVe1 exhibit enhanced resistance to Verticillium dahliae. Plant Mol. Biol. Rep. 30:1-8.

Liu, Y. L., Schiff, M., and Dinesh-Kumar, S. P. 2002. Virus-induced gene silencing in tomato. Plant J. 31:777-786.

Nakagawa, T., Suzuki, T., Murata, S., Nakamura, S., Hino, T., Maeo, K. Tabata, R., Kawai, T., Tanaka, K., Niwa, Y., Watanabe, Y., Nakamura, K., Kimura, T., and Ishiguro, S. 2007. Improved gateway binary vectors: High-performance vectors for creation of fusion constructs in transgenic analysis of plants. Biosci. Biotechnol. Biochem. 71:20952100.

Paternotte, S. J., and van Kesteren, H. A. 1993. A new aggressive strain of Verticillium albo-atrum in Verticillium resistant cultivars of tomato in the Netherlands. Neth. J. Plant Pathol. 99:169-172.

Rowland, O., Ludwig, A. A., Merrick, C. J., Baillieul, F., Tracy, F. E., Durrant, W. E., Fritz-Laylin, L., Nekrasov, V., Sjolander, K., Yoshioka, H., and Jones, J. D. G. 2005. Functional analysis of Avr9/Cf-9 rapidly elicited genes identifies a protein kinase, ACIK1, that is essential for full Cf-9-dependent disease resistance in tomato. Plant Cell 17:295310

Ryu, C. M., Anand, A., Kang, L., and Mysore, K. S. 2004. Agrodrench: A novel and effective agroinoculation method for virus-induced gene silencing in roots and diverse Solanaceous species. Plant J. 40:322-331.

Sainsbury, F., Thuenemann, E. C., and Lomonossoff, G. P. 2009. pEAQ: Versatile expression vectors for easy and quick transient expression of heterologous proteins in plants. Plant Biotechnol J. 7:682-693.

Santos-Rosa, M., Poutaraud, A., Merdinoglu, D., and Mestre, P. 2008. Development of a transient expression system in grapevine via agro-infiltration. Plant Cell Rep. 27:1053-1063.

Senthil-Kumar, M., Govind, G., Kang, L., Mysore, K. S., and Udayakumar, M. 2007. Functional characterization of Nicotiana benthamiana homologs of peanut water deficit-induced genes by virus-induced gene silencing. Planta 225:523-539.

Shimada, T. L., Shimada, T., and Hara-Nishimura, I. 2010. A rapid and non-destructive screenable marker, FAST, for identifying transformed seeds of Arabidopsis thaliana. Plant J. 61:519-528.

Simko, I., Haynes, K. G., Ewing, E. E., Costanzo, S., Christ, B. J., and Jones, R. W. 2004. Mapping genes for resistance to Verticillium alboatrum in tetraploid and diploid potato populations using haplotype association tests and genetic linkage analysis. Mol. Genet. Genomics 271:522-531.
Stulemeijer, I. J. E., Stratmann, J. W., and Joosten, M. H. A. J. 2007. Tomato mitogen-activated protein kinases LeMPK1, LeMPK2, and LeMPK3 are activated during the Cf-4/Avr4-induced hypersensitive response and have distinct phosphorylation specificities. Plant Physiol. 144:14811494.

Thomma, B. P. H. J., van Esse, H. P., Crous, P. W., and De Wit, P. J. G. M. 2005. Cladosporium fulvum (syn. Passalora fulva), a highly specialized plant pathogen as a model for functional studies on plant pathogenic Mycosphaerellaceae. Mol. Plant Pathol. 6:379-393.

Thomma, B. P. H. J., Nurnberger, T., and Joosten, M. H. A. J. 2011. Of PAMPs and effectors: The blurred PTI-ETI dichotomy. Plant Cell 23:415

van der Gheynst, J. S., Guo, H. Y., and Simmons, C. W. 2008. Response surface studies that elucidate the role of infiltration conditions on Agrobacterium tumefaciens-mediated transient transgene expression in harvested switchgrass (Panicum virgatum). Biomass Bioenerg. 32:372-379.

Van der Hoorn, R. A. L., Laurent, F., Roth, R., and De Wit, P. J. G. M. 2000. Agroinfiltration is a versatile tool that facilitates comparative analyses of Avr $9 / C f$-9-induced and Avr4/Cf-4-induced necrosis. Mol. Plant-Microbe Interact. 13:439-446.

Van der Hoorn, R. A., Rivas, S., Wulff, B. B., Jones, J. D., and Joosten, M H. 2003. Rapid migration in gel filtration of the Cf-4 and Cf-9 resistance proteins is an intrinsic property of $\mathrm{Cf}$ proteins and not because of their association with high-molecular-weight proteins. Plant J. 35:305-315.

Velasquez, A. C., Chakravarthy, S., and Martin, G. B. 2009. Virus-induced gene silencing in Nicotiana benthamiana and tomato. J. Vis. Exp. 10:1292

Vining, K., and Davis, T. 2009. Isolation of a Ve homolog, $m V e 1$, and its relationship to Verticillium wilt resistance in Mentha longifolia L. Huds. Mol. Genet. Genomics 282:173-184.

Vining, K. J., Zhang, Q., Smith, C. A., and Davis, T. M. 2007. Identification of resistance gene analogs and Verticillium wilt resistance-like sequences in Mentha longifolia. J. Am. Soc. Hortic. Sci. 132:541-550.

Voinnet, O., Rivas, S., Mestre, P., and Baulcombe, D. 2003. An enhanced transient expression system in plants based on suppression of gene silencing by the 19 protein of tomato bushy stunt virus. Plant J. 33:949-956.

Vossen, J. H., Abd-El-Haliem, A., Fradin, E. F., van den Berg, G. C. M., Ekengren, S. K., Meijer, H. J. G., Seifi, A., Bai, Y. L., ten Have, A. Munnik, T., Thomma, B. P. H. J., and Joosten, M. H. A. J. 2010. Identification of tomato phosphatidylinositol-specific phospholipase-C (PIPLC) family members and the role of PLC4 and PLC6 in HR and disease resistance. Plant J. 62:224-239.

Wang, G., Ellendorff, U., Kemp, B., Mansfield, J. W., Forsyth, A., Mitchell, K., Bastas, K., Liu, C. M., Woods-Tor, A., Zipfel, C., de Wit, P. J. G. M., Jones, J. D., Tor, M., and Thomma, B. P. H. J. 2008. A genome-wide functional investigation into the roles of receptor-like proteins in Arabidopsis. Plant Physiol. 147:503-517.

Wang, G., Fiers, M., Ellendorff, U., Wang, Z., de Wit, P. J. G. M., Angenent, G., and Thomma, B. P. H. J. 2010. The diverse roles of extracellular leucine-rich repeat-containing receptor-like proteins in plants. Crit. Rev. Plant Sci. 29:285-299.

Wang, Y. Z., Tzfira, T., Gaba, V., Citovsky, V., Palukaitis, P., and Gal-On, A. 2004. Functional analysis of the Cucumber mosaic virus $2 b$ protein: Pathogenicity and nuclear localization. J. Gen. Virol. 85:3135-3147.

Wroblewski, T., Tomczak, A., and Michelmore, R. 2005. Optimization of Agrobacterium-mediated transient assays of gene expression in lettuce, tomato and Arabidopsis. Plant Biotechnol. J. 3:259-273.

Wulff, B. B. H., Heese, A., Tomlinson-Buhot, L., Jones, D. A., de la Pena, M., and Jones, J. D. G. 2009. The major specificity-determining amino acids of the tomato $\mathrm{Cf}-9$ disease resistance protein are at hypervariable solvent-exposed positions in the central leucine-rich repeats. Mol. Plant-Microbe Interact. 22:1203-1213.

Zhang, Y., Wang, X., Yang, S., Chi, J., Zhang, G., and Ma, Z. 2011. Cloning and characterization of a Verticillium wilt resistance gene from Gossypium barbadense and functional analysis in Arabidopsis thaliana. Plant Cell Rep. 30:2085-2096. 